\title{
Einlaufverhalten nasslaufender Lamellenkupplungen im Schlupfbetrieb
}

\author{
Katharina Völkel' ${ }^{1 D} \cdot$ Hermann Pflaum ${ }^{1}$ (D) $\cdot$ Karsten Stahl' ${ }^{1}$ (ID \\ Eingegangen: 11. Juni 2021 / Angenommen: 28. Juli 2021 / Online publiziert: 23. November 2021 \\ (c) Der/die Autor(en) 2021
}

\section{Zusammenfassung}

Nasslaufende Lamellenkupplungen weisen zu Betriebsbeginn eine Phase des Einlaufens auf, in der sich das Reibungsverhalten durch Anpassung der Reibflächen signifikant ändern kann. Dieses Einlaufverhalten ist in Anhängigkeit der Charakteristika des Reibsystems bestehend aus Reibbelag, Stahllamelle und Schmierstoff stark unterschiedlich ausgeprägt. Mit dem Ziel einer guten Regelbarkeit und damit hohen Zuverlässigkeit der Kupplung ab der ersten Schaltung sollen Einlaufvorgänge wenig ausgeprägt und schnell abgeschlossen sein.

Die Charakterisierung des Einlaufverhaltens im Schlupfbetrieb erfolgt experimentell an Kupplungen mit Carbonreibbelag aus den Anwendungen Verteilergetriebe und Sperrdifferential im Instationärschlupf. Eine neu entwickelte Versuchsmethode erlaubt es, das Einlaufverhalten für mehrere anwendungstypische Lastniveaus zu untersuchen. Relevante Lastniveaus werden aus Fahrzeugmessungen abgeleitet. Des Weiteren wird eine Auswertemethode vorgestellt, die über die Definition spezifischer Kennwerte für Schlupfbetrieb eine quantitative Beurteilung von Ausprägung und Dauer der Einlaufvorgänge realisiert. Das Einlaufverhalten von Kupplungen mit unterschiedlichen Reibsystemen wird im Zuge einer Systembewertung gegenübergestellt, Einflüsse von Schmierstoff, Stahllamelle und Reibbelag sowie Wechselwirkungen werden systematisch charakterisiert.

\section{Running-in behavior of wet multi-plate clutches in slip operation mode}

\begin{abstract}
Wet multi-plate clutches have a running-in phase at the start of operation in which the friction behavior can change significantly by adjusting the friction surfaces. This running-in behavior can strongly depend on the characteristics of the friction system consisting of friction material, steel plate and fluid. With the aim of good controllability and thus high reliability of the clutch from the first shift, running-in processes should be less pronounced and should be completed quickly.

The characterization of the running-in behavior in slip operation mode is carried out experimentally on clutches with carbon friction lining from the applications transfer case and limited slip differential in transient slip. A newly developed test method allows examining the running-in behavior for several application-typical load levels. Relevant load levels are derived from vehicle measurements. Furthermore, an evaluation method is presented that realizes a quantitative assessment of the characteristics and duration of the running-in processes by defining new parameters for slip operation mode. The running-in behavior of clutches with different friction systems is compared in the course of a system evaluation, Influences of lubricant, steel plate and friction lining as well as interactions are systematically characterized.
\end{abstract}

Availability of data and material The data that support the findings of this study are available from the Research Association for Drive Technology e. V. (FVA) but restrictions apply to the availability of these data, which were used under license for the current study, and so are not publicly available. Data are however available from the authors upon reasonable request and with permission of FVA.

Code availability Not applicable.
Katharina Völkel

voelkel@fzg.mw.tum.de

1 Forschungsstelle für Zahnräder und Getriebebau (FZG), Technische Universität München, Boltzmannstr. 15, 85748 Garching b. München, Deutschland 


\section{Einleitung}

In modernen Antriebssträngen stellen nasslaufende Lamellenkupplungen funktions- und sicherheits- sowie komfortrelevante Komponenten dar. So werden nasslaufende Lamellenkupplungen beispielsweise in Sperren von aktiven Längs- und Querdifferenzialen verwendet. HinterachsSperrdifferentiale realisieren eine hohe Sperrwirkung typischerweise durch den Einsatz nasslaufender Lamellenkupplungen.

Ziele von Forschung und Entwicklung sind ein stabiles und hohes Reibungszahlniveau bei gleichzeitig günstiger Reibcharakteristik (Verlauf der Reibungszahl über der Gleitgeschwindigkeit), um ein gutes Übertragungsverhalten bei gleichzeitig hohem Schaltkomfort zu realisieren (vgl. z.B. [1]).

Insbesondere zu Lebensdauerbeginn, wenn Einlaufvorgänge noch nicht abgeschlossen sind, kann sich das Reibungsverhalten durch Einglätten und Anpassen der Reibpaarung sowie den Aufbau chemischer Grenzschichten signifikant ändern (vgl. z. B. [2-4]). Dieses Einlaufverhalten ist in Abhängigkeit der Charakteristika des tribologischen Systems, bestehend aus den im Reibkontakt befindlichen Reibpartnern sowie dem Schmierstoff und den Betriebsbedingungen, stark unterschiedlich ausgeprägt (vgl. z.B. [5-8]).

Mit dem Ziel einer guten Regelbarkeit und damit hohen Zuverlässigkeit der Kupplung ab der ersten Schaltung stehen zwei Punkte im Vordergrund der Entwicklungen. Zum einen sollen Einlaufvorgänge, also Änderungen des Reibungsverhaltens zu Betriebsbeginn, möglichst wenig ausgeprägt und schnell abgeschlossen sein. Zum anderen soll die Kupplung bereits im Einlauf ein gutes Reibungsverhalten (Reibungszahlniveau und Reibcharakteristik) aufweisen.

Im Stand des Wissens wird das Ende des Einlaufs typisch mit dem Erreichen eines stabilen Reibungsverhaltens gleichgesetzt (vgl. z. B. [9-11]). In den Versuchen zur Entwicklung der Methodik „Einlaufverhalten Lamellenkupplung" [12] treten zu Versuchsbeginn stets deutliche, nichtkonstante Änderungen im Reibungsverhalten auf. Im weiteren Versuchsverlauf werden diese Änderungen im Reibungsverhalten konstant, d.h. der Trendverlauf nimmt einen linearen Verlauf an. Typisch ist die Steigung der Trendverläufe nach dem Einlauf sehr gering. Anhand von stichprobenartig durchgeführten, länger laufenden Versuchen ist erkennbar, dass der Trendverlauf mit zunehmender Versuchslaufzeit ein näherungsweise konstantes Reibungszahlniveau annimmt. In Zusammenarbeit mit Experten aus der Industrie wird erarbeitet, dass insbesondere nichtkonstante Änderungen im Reibungsverhalten, also ein nichtlinearer Trendverlauf, eine sehr große Herausforderung für die Kupplungsansteuerung darstellen, während konstante Änderungen des Reibungsverhaltens, also ein linearer Trend- verlauf, für den Kupplungscontroller erlernbar sind. Damit ist aus Anwendungssicht der Einlauf einer Kupplung - im Sinne eines instabilen Zustands - durch einen nichtlinearen Trend des Reibungsverhaltens gekennzeichnet. Der Einlauf gilt als abgeschlossen, wenn der Reibungszahltrend einen näherungsweise linearen Verlauf annimmt.

\section{Methoden und Versuchsteile}

In [12] wird auf Basis definierter Anforderungen eine Methodik „Einlaufverhalten Lamellenkupplungen“ zur Charakterisierung des Einlaufverhaltens nasslaufender Lamellenkupplungen entwickelt. Die Methodik umfasst Versuchsund Auswertemethoden.

\subsection{Versuchsmethode}

Die Versuchsmethode für Kupplungen im Schlupfbetrieb wird aus der bereits in [13] veröffentlichten Versuchsmethode für lastschaltende Kupplungen abgeleitet. Mit dem Ziel, das Reibungsverhalten schlupfender Kupplungen möglichst anwendungsnah zu untersuchen, wird als Betriebsart Instationärschlupf (vgl. Abb. 1) gewählt.

Analog zur Versuchsmethode für lastschaltende Kupplungen wird auch für Instationärschlupf ein Lastkollektiv bestehend aus je einem Schlupfzyklus auf verschiedenen anwendungstypischen Laststufen entwickelt (vgl. Abb. 2). So kann das Einlaufverhalten auf mehreren anwendungsnahen Lastniveaus charakterisiert werden. Um die spezifischen Belastungen der Kupplungen im Versuch möglichst anwendungsnah zu wählen, werden Fahrzeugdaten aus verschiedenen Testfahrten (Rennstrecken, Passstraßen, $\mu$-split) ausgewertet und relevante Lastzustände identifiziert. Das Vorgehen ist in [12] umfassend beschrieben. Zudem finden die Produktspezifikationen der verwendeten Reibbeläge Berücksichtigung.

Das Lastkollektiv wird zyklisch wiederholt, bis der Einlauf sicher abgeschlossen ist (hier 250 Kollektivwiederholungen).

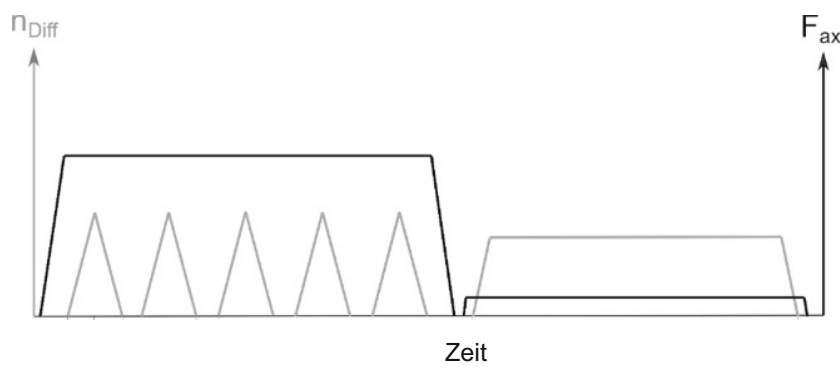

Abb. 1 Schematische Darstellung eines Schlupfzyklus im Instationärschlupf [12] 
Abb. 2 Lastkollektiv für schlupfende Kupplungen - schematische Darstellung des Kollektivs (a) und spezifische Belastungen in den Laststufen (b) [12, $14]$ a

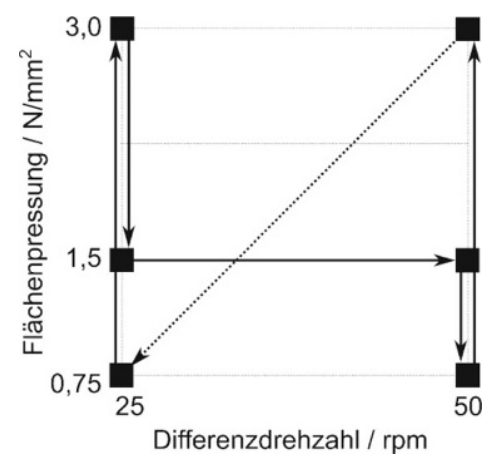

b

\begin{tabular}{|l|l|l|}
\hline Laststufe & $\begin{array}{l}\text { Flächenpressung } / \text { | } \\
\mathbf{N} / \mathbf{m m}^{\mathbf{2}}\end{array}$ & $\begin{array}{l}\text { DifferenzdrehzahI } / \\
\mathbf{m i n}^{-1}\end{array}$ \\
\hline S1 & 0,75 & 25 \\
\hline S2 & 1,50 & 25 \\
\hline S3 & 3,00 & 25 \\
\hline S4 & 0,75 & 50 \\
\hline S5 & 1,50 & 50 \\
\hline S6 & 3,00 & 50 \\
\hline
\end{tabular}

\subsection{Auswertemethoden}

Die Auswertung des Einlaufverhaltens erfolgt in mehreren Schritten. Ein grundlegender und wichtiger Schritt besteht in einer umfassenden Betrachtung der Reibcharakteristiken sowie der Identifikation charakterisierender Reibungszahlkennwerte. Im Schlupfbetrieb werden die neu entwickelten Reibungszahlkennwerte $\mu_{\text {top }}$ und $\mathrm{m}_{\mu}$ herangezogen [12, 13]. $\mu_{\text {top }}$ beschreibt hierbei die Reibungszahl bei maximaler Gleitgeschwindigkeit $\left(100 \% \mathrm{v}_{\mathrm{g}, \max }\right)$ und charakterisiert damit das Reibungszahlniveau. Der Kennwert $\mathrm{m}_{\mu}$ quantifiziert die Steigung einer Ausgleichsgeraden (lineare Regression nach der Methode der kleinsten Quadrate) im Gleitgeschwindigkeitsbereich $30 \ldots 80 \% \mathrm{v}_{\mathrm{g} \text {,max }}$ und charakterisiert somit die Reibschwingneigung. Abb. 3 zeigt exemplarische Messsignalverläufe von Axialkraft $\mathrm{F}_{\mathrm{A}}$, Drehmoment $\mathrm{T}_{\mathrm{R}}$, Drehzahl $n$ und errechneter Reibungszahl $\mu$ (Abb. 3a) und die zugehörigen Reibcharakteristiken der fallenden und steigenden Flanke des fünften Schlupfzyklus samt den Werten für $\mu_{\text {top }}$ und $\mathrm{m}_{\mu}$ (Abb. 3b). a

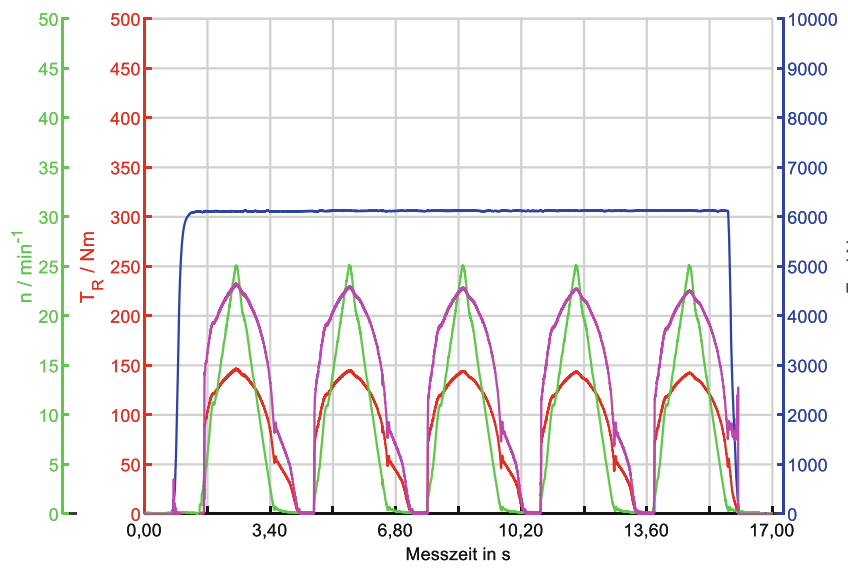

Die Reibungszahlkennwerte werden in Reibungszahltrends über der Schaltungszahl aufgetragen, um die Entwicklung des Reibungsverhaltens zu visualisieren. Das Ende des Einlaufs wird mittels mathematischer Methoden identifiziert; [12] stellt hierzu zwei verschiedene mathematische Methoden vor. Im vorliegenden Beitrag werden die Ergebnisse mittels der Methode der linearen Regressionsanalyse ermittelt, da diese aufgrund ihrer breiten Anwendbarkeit eine hohe Anwendungsorientierung aufweist. Hierbei wird beginnend mit den Reibungszahlkennwerten der letzten zwanzig Kollektivdurchläufe des Versuchs (hier 231 bis 250) eine lineare Regressionsanalyse durchgeführt und die Messwerte dieser zwanzig Kollektivdurchläufe durch eine parametrierbare Geradengleichung (1) beschrieben; dann werden die Residuen, also Abweichungen der Einzelmesswerte von der Ausgleichsgeraden, aller vorherigen Kollektivdurchläufe (hier 1 bis 230) bestimmt. Im nächsten Schritt wird für die Bestimmung der Geradengleichung der letzte vorhergehende Kollektivdurchlauf (hier 230, dann 229, usw.) hinzugezogen, sodass die Basis der Geraden größer wird. Dies wird iterativ durchlaufen, bis

Abb. 3 Messschrieb (a) und Reibcharakteristik der fünften Schlupfphase (b) [12]

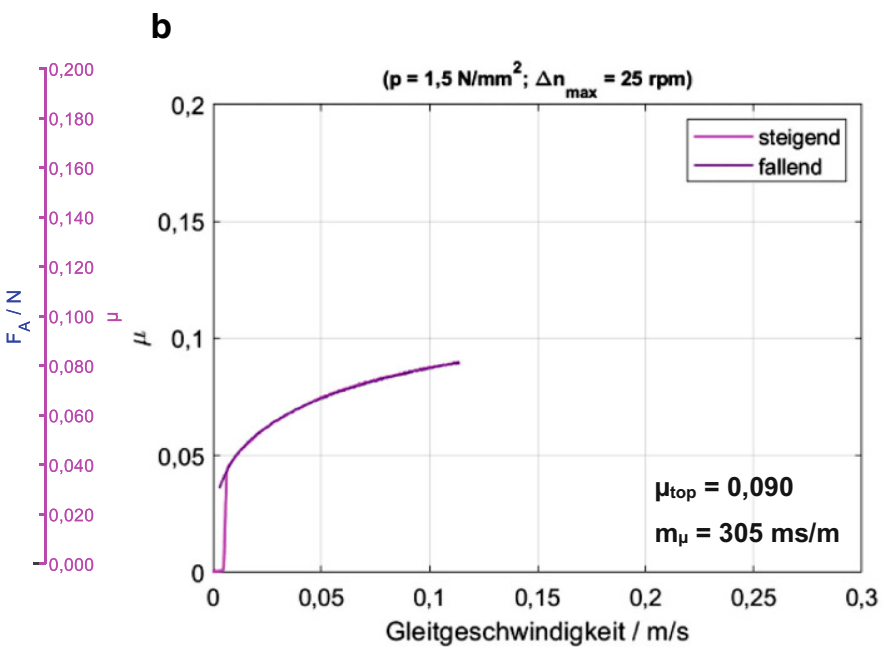

\footnotetext{
Abb. 3 Messschrieb (a) und Reibcharakteristik der funften Schlupfphase (b) [12]
} 
Abb. 4 Trendverlauf $\mu_{\text {top }}$ - Einzelmesswerte und Ausgleichsgerade mit charakterisierenden Kennwerten $\mathrm{X}_{\text {lin }}$ und $\Delta \mu_{\text {start }}[12]$

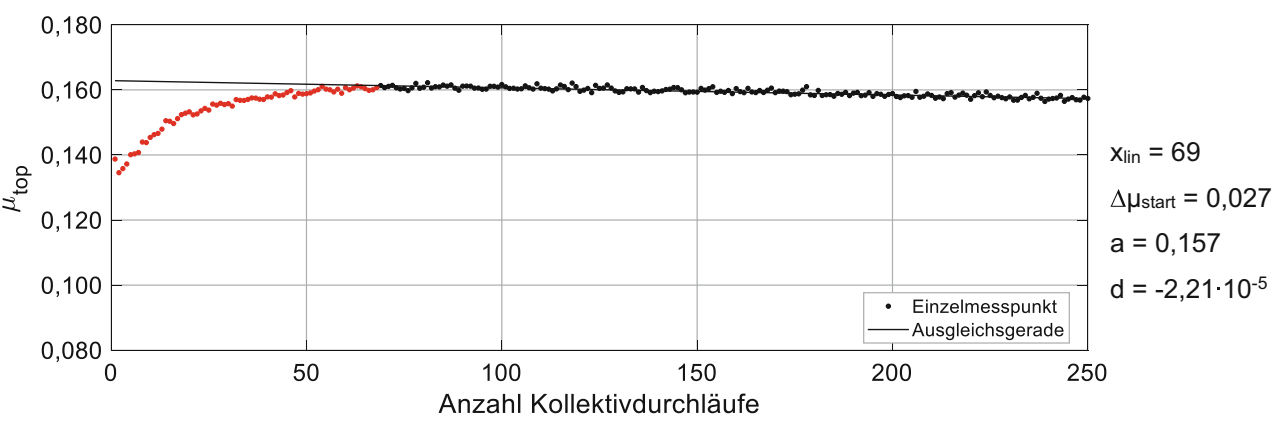

alle Residuen der vorher gefahrenen Kollektivdurchläufe das gleiche Vorzeichen aufweisen, also entweder ober- oder unterhalb der Regressionsgeraden liegen. Ist dieses Abbruchkriterium erreicht, so ist der Übergang vom nichtlinearen Bereich des Trendverlaufs in den linearen und damit das Ende des Einlaufs gefunden.

$\mu_{\text {top }}=\mathrm{f}(\mathrm{x})=\mathrm{a}+\mathrm{d} \cdot(\mathrm{x}-\mathrm{n})$

Regressionsparameter, Reibungszahlniveau zu Versuchsende

d Regressionsparameter, Steigung der Ausgleichsgeraden

n

Anzahl Kollektivdurchläufe im Versuch

Neu entwickelte Kennwerte charakterisieren das Einlaufverhalten: Der Kennwert $\mathrm{x}_{\text {lin }}$ gibt die Dauer des Einlaufs an; der Kennwert $\Delta \mu_{\text {start }}$ beschreibt die maximale Reibungszahländerung im Einlauf.

In Abb. 4 umfasst die Dauer des Einlaufs $x_{\text {lin }}=69$ Kollektivdurchläufe; die Reibungszahländerung im Einlauf beträgt $\Delta \mu_{\text {start }}=0,027$.

Die Kennwerte $\Delta \mu_{\text {start }}$ und $\mathrm{x}_{\text {lin }}$ quantifizieren das Einlaufverhalten. Bei einer Gegenüberstellung mehrerer Reibsysteme ist es das Ziel, deren Einlaufverhalten anhand eines
Kennwerts ganzheitlich bewerten zu können. In [12] wird deshalb der Kennwert Einlauf-Systembewertung EW entwickelt, der die charakterisierenden Kennwerte $\Delta \mu_{\text {start }}$ und $\mathrm{x}_{\text {lin }}$ zusammenführt. Zur Ermittlung des Kennwerts werden die charakterisierenden Kennwerte $\Delta \mu_{\text {start }}$ und $\mathrm{x}_{\text {lin }}$ normiert, indem diese ins Verhältnis zu Bezugsgrößen $\Delta \mu_{\text {start,N }}$ und $\mathrm{x}_{\mathrm{lin}, \mathrm{N}}$ gesetzt werden (vgl. Formeln (2) und (3)). Diese Bezugsgrößen können z. B. Sollanforderungen (aus Praxisfunktionalität), der IST-Zustand des derzeit in Serie befindlichen Systems oder Mittelwerte über alle betrachteten Reibsysteme sein. Die so berechneten, normierten Kennwerte $\Delta \mu_{\text {start }}^{*}$ und $\mathrm{x}_{\text {lin }}^{*}$ zum Einlaufverhalten werden gemäß Formel (4) mit den Faktoren $\mathrm{q}_{1}$ und $\mathrm{q}_{2}$ gewichtet und in den Kennwert Einlauf-Systembewertung EW zur ganzheitlichen Bewertung des Einlaufverhaltens überführt. Ein System mit identischem Einlaufverhalten wie das Bezugssystem hat hierbei einen Wert EW von 0. Weist das betrachtete System ein besseres Einlaufverhalten als das Bezugssystem auf, ist der Wert EW positiv, bei einem schlechteren Einlaufverhalten ist der Wert EW negativ. Formel (5) gibt die mathematischen Grenzen der Gewichtungsfaktoren vor.

$$
\begin{aligned}
& \Delta \mu_{\text {start }}^{*}=\frac{\Delta \mu_{\text {start }}}{\Delta \mu_{\text {start }, \mathrm{N}}} \\
& \mathrm{x}_{\text {lin }}^{*}=\frac{\mathrm{x}_{\text {lin }}}{\mathrm{x}_{\text {lin }, \mathrm{N}}}
\end{aligned}
$$

Abb. 5 Kupplungen in Baugröße BCI (a) und BCII (b) Stahllamelle und Belaglamellen a

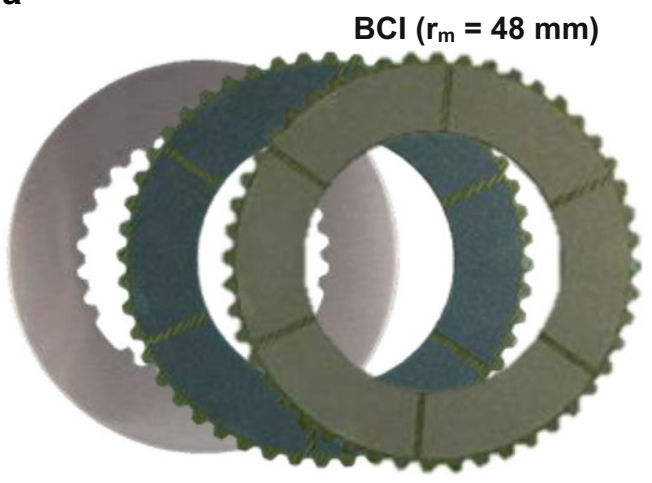

b

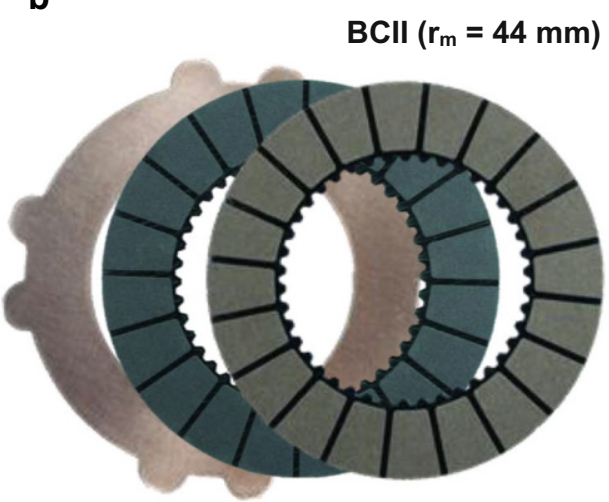


Abb. 6 Einlauf-Systembewertung - Einflüsse des Schmierstoffs auf das Einlaufverhalten (Baugröße BCI) [12]
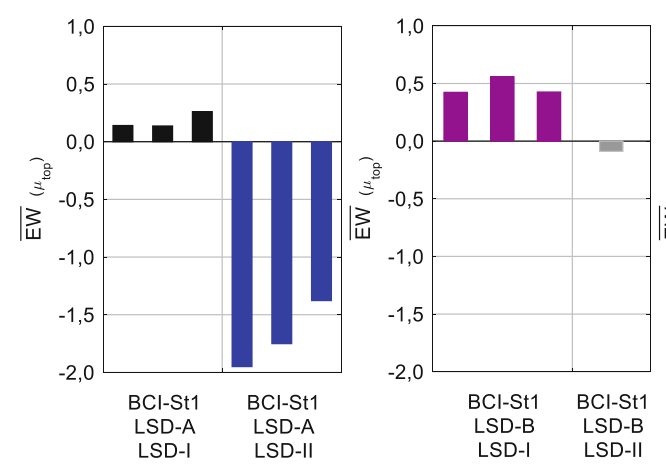
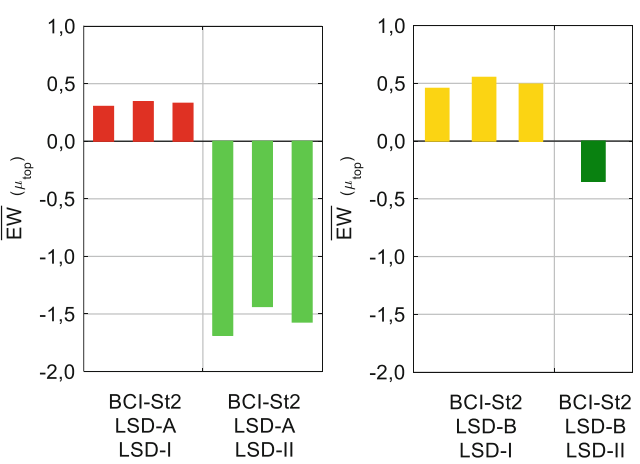

$\mathrm{EW}=1-\left(\mathrm{q}_{1} \cdot \Delta \mu_{\text {start }}^{*}+\mathrm{q}_{2} \cdot \mathrm{x}_{\text {lin }}^{*}\right)$

$\mathrm{q}_{1}+\mathrm{q}_{2}=1$ mit $0<\mathrm{q}_{1}<1$ und $0<\mathrm{q}_{2}<1$

\begin{tabular}{|c|c|}
\hline $\mathrm{X}_{\text {lin }}$ & $\begin{array}{l}\text { Kennwert zum Einlaufverhalten, Dauer } \\
\text { des Einlaufs }\end{array}$ \\
\hline$\Delta \mu_{\text {start }}$ & $\begin{array}{l}\text { Regressionsparameter, Steigung der Aus- } \\
\text { gleichsgeraden }\end{array}$ \\
\hline$\Delta \mu_{\text {start }}^{*}, x_{\text {lin }}^{*}$ & $\begin{array}{l}\text { normierte Kennwerte zum Einlaufverhal- } \\
\text { ten }\end{array}$ \\
\hline$\Delta \mu_{\text {start }, \mathrm{N}}, \mathrm{X}_{\mathrm{lin}, \mathrm{N}}$ & $\begin{array}{l}\text { Bezugsgrößen (Sollanforderungen, IST- } \\
\text { Zustand, Mittelwerte,...) }\end{array}$ \\
\hline EW & $\begin{array}{l}\text { Kennwert zum Einlaufverhalten, Einlauf- } \\
\text { Systembewertung }\end{array}$ \\
\hline $1, q 2$ & Gewichtungsfaktoren \\
\hline
\end{tabular}

Analog der Kennwerte $\Delta \mu_{\text {start }}$ und $\mathrm{x}_{\text {lin }}$ sind die Werte EW für jede Laststufe des Lastkollektivs zu bestimmen. Um das Einlaufverhalten unterschiedlicher Reibsysteme gegenüberzustellen, wird in der vorliegenden Arbeit der Mittelwert $\overline{\mathrm{EW}}$ über die Werte EW aller Laststufen gebildet.

\subsection{Versuchsteile und Schmierstoffe}

Die Untersuchungen werden an Kupplungen in zwei Baugrößen BCI und BCII aus den Anwendungsfeldern Verteilergetriebe und Sperrdifferential durchgeführt. Es erfolgt eine Variation der Stahllamellenbeschaffenheit, des Reibbelags und des Schmierstoffs. Hierbei kommen je zwei typische Stahllamellenvarianten, Reibbeläge und Schmierstoffe
Abb. 7 Einlauf-Systembewertung - Einflüsse des Reibbelags auf das Einlaufverhalten (Baugröße BCI) [12]
Abb. 8 Einlauf-Systembewertung - Einflüsse der Stahllamellenbeschaffenheit auf das Einlaufverhalten (Baugröße BCI) [12]
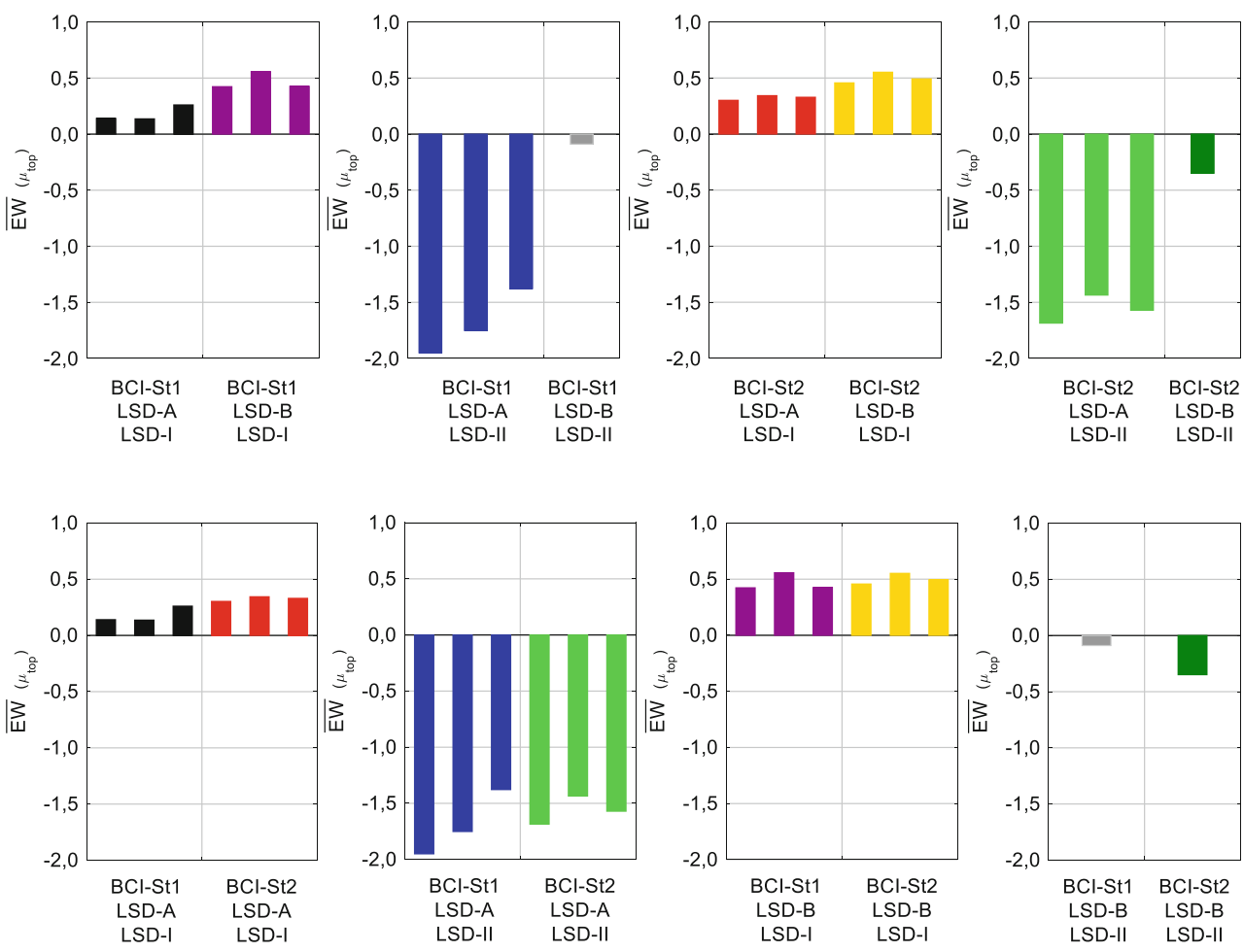
Tab. 1 Stahllamellenvarianten

\begin{tabular}{lll}
\hline BCI & BCI-St1 & Bandgeschliffen I Serienstahllamelle \\
& $B C I-S t 2$ & Bandgeschliffen und gebürstet \\
BCII & $B C I I-S t 1$ & $\begin{array}{l}\text { Bandgeschliffen und gebürstet I Serienstahlla- } \\
\text { melle }\end{array}$ \\
& $B C I I-S t 2$ & Bandgeschliffen \\
\hline
\end{tabular}

Tab. 2 Schmierstoffe - technische Daten [12]

\begin{tabular}{llll}
\hline & Einheit & LSD-I & LSD-II \\
\hline Dichte bei $15^{\circ} \mathrm{C}$ & $\mathrm{Kg} / \mathrm{m}^{3}$ & 863 & 879 \\
Kinematische Viskosität bei $100^{\circ} \mathrm{C}$ & $\mathrm{mm}^{2} / \mathrm{s}$ & 9,0 & 14,9 \\
Kinematische Viskosität bei $40^{\circ} \mathrm{C}$ & $\mathrm{mm}^{2} / \mathrm{s}$ & 46,5 & 81,0 \\
\hline
\end{tabular}

zum Einsatz. Abb. 5 zeigt Fotos der Stahl- und Belaglamellen mit Angabe der mittleren Reibradien $r_{m}$.

Tab. 1 führt die Stahllamellenvarianten beider Baugrößen auf.

Die Reibbeläge sind Carbonreibbeläge und tragen die Bezeichnungen LSD-A und LSD-B. Reibbelag LSD-A ist Serienbelag in der Baugröße BCI, LSD-B in der Baugröße BCII. Die Reibbeläge wurden auch auf die Serienbelagträger der jeweils anderen Baugröße aufgebracht.

Als Schmierstoffe werden typische Serienöle aus den Anwendungen Sperrdifferential (LSD-I) und Verteilergetriebe (LSD-II) verwendet. In Tab. 2 sind die technischen Daten der Schmierstoffe gemäß Datenblatt zusammengestellt.

\section{Ergebnisse}

Die Einlauf-Systembewertung EW ermöglicht eine ganzheitliche Charakterisierung der Systemeigenschaften hinsichtlich des Einlaufverhaltens. Die systematische Auswertung von Einflüssen des Reibsystems auf das Einlaufverhalten erfolgt deshalb im Folgenden anhand dieses Kennwerts. Im Rahmen dieser Veröffentlichung wird das Einlaufverhalten bezogen auf den Reibungszahlkennwert $\mu_{\text {top }}$ betrachtet. Auswertungen des Reibungszahlkennwerts $m_{\mu}$ sind in [12] aufgeführt. Auch bezüglich dieses Kennwerts zeigen sich deutliche Einflüsse des Reibsystems.

Als Bezugsgrößen $\Delta \mu_{\text {start, } N}$ und $x_{\text {lin, } N}$ werden Mittelwerte aus den Versuchen mit Baugröße BCI und BCII herangezogen. Da nicht immer die gleiche Anzahl Versuche je Reibsystem durchgeführt wurde, wird zunächst für jedes Reibsystem ein Mittelwert aus allen Versuchen dieses Reibsystems gebildet. Diese hier acht Mittelwerte (vier je Baugröße) gehen dann in die Ermittlung der Bezugsgrößen $\Delta \mu_{\text {start,N }}$ und $\mathrm{x}_{\text {lin,N }}$ ein. Tab. 3 führt die Bezugsgrößen $\Delta \mu_{\text {start }, N}$ und $\mathrm{x}_{\text {lin,N }}$ für die Laststufen $\mathrm{S} 1$ bis S6 auf. Es zeigt sich eine teils deutliche Abhängigkeit von der spezifischen Belastung
Tab. 3 Bezugsgrößen $\Delta \mu_{\text {start,N }}$ und $\mathrm{X}_{\text {lin,N }}$ aus Mittelwertbildung über alle Versuche der Baugrößen BCI und BCII [12]

\begin{tabular}{lllllll}
\hline & $\mathrm{S} 1$ & $\mathrm{~S} 2$ & $\mathrm{~S} 3$ & $\mathrm{~S} 4$ & $\mathrm{~S} 5$ & $\mathrm{~S} 6$ \\
\hline$\Delta \mu_{\text {start }, N}$ & 0,011 & 0,008 & 0,011 & 0,007 & 0,007 & 0,009 \\
$X_{\text {lin }, N}$ & 25 & 36 & 44 & 24 & 36 & 48 \\
\hline
\end{tabular}

Tab. 4 Einflüsse des Reibsystems auf das Einlaufverhalten in Versuchen mit Kupplungen BCI (Verteilergetriebe) und BCII (Sperrdifferential)

\begin{tabular}{lll}
\hline & Verteilergetriebe (BCI) & Sperrdifferential (BCII) \\
\hline Stahllamelle & $\bigcirc$ schwacher Einfluss & $\bullet$ starker Einfluss \\
Reibbelag & $\ominus$ moderater Einfluss & $\ominus$ moderater Einfluss \\
Schmierstoff & $\bullet$ starker Einfluss & $\ominus$ moderater Einfluss \\
\hline
\end{tabular}

in den unterschiedlichen Laststufen. $\mathrm{x}_{\text {lin }}$ wird mit $\mathrm{q}_{2}=20 \%$ gewichtet, die Gewichtung des Parameters $\Delta \mu_{\text {start }}$ beträgt damit $\mathrm{q}_{1}=80 \%$; die Werte können anwendungsspezifisch abweichend gewählt werden.

Die folgenden Bilder zeigen die Mittelwerte $\overline{\mathrm{EW}}$ für die Versuche mit Baugröße BCI in drei unterschiedlichen Gruppierungen, um die Einflüsse des Schmierstoffs (Abb. 6), des Reibbelags (Abb. 7) und der Stahllamellenbeschaffenheit (Abb. 8) darzustellen. Hierbei entspricht jeder Balken einem Versuch mit jeweils unterschiedlichem Lamellenpaket. Es werden bis zu drei Versuche mit Kupplungspaketen eines Reibsystems durchgeführt, um die Reproduzierbarkeit der Ergebnisse sicherzustellen.

Der Schmierstoff hat in den Versuchen mit Kupplungen der Baugröße BCI einen sehr deutlichen Einfluss auf das Einlaufverhalten. Versuche mit Schmierstoff LSD-I weisen in allen Stahllamellen-Reibbelag-Kombinationen positive Werte $\overline{\mathrm{EW}}$ auf, während die Werte $\overline{\mathrm{EW}}$ von Versuchen mit Schmierstoff LSD-II negativ sind. Zudem besteht eine Wechselwirkung zum Reibbelag: In Versuchen mit Reibbelag LSD-A sind Unterschiede zwischen den beiden Schmierstoffen deutlicher ausgeprägt als mit Reibbelag LSD-B. In Versuchen mit Reibbelag LSD-B zeigt sich zudem eine Wechselwirkung zwischen Schmierstoff und Stahllamellenbeschaffenheit. Der Einfluss des Schmierstoffs ist bei diesem Reibbelag mit Stahllamellen BCI-St1 deutlicher als mit Stahllamellen BCI-St2.

Einflüsse des Reibbelags auf das Einlaufverhalten sind ebenfalls deutlich zu erkennen. Der Reibbelag LSD-B zeigt in allen Versuchen mit Kupplungen der Baugröße BCI höhere Werte $\overline{\mathrm{EW}}$ und damit das bessere Einlaufverhalten als Versuche mit Reibbelag LSD-A bei gleicher Stahllamellen-Schmierstoff-Kombination. Die Wechselwirkung zwischen Reibbelag und Schmierstoff tritt deutlich hervor, da Unterschiede im Einlaufverhalten der Reibbeläge LSD-A und LSD-B mit Schmierstoff LSD-II deutlicher ausgeprägt sind als mit Schmierstoff LSD-I. Wechselwirkungen mit der Stahllamellenvariante sind nicht eindeutig erkennbar. 
Einflüsse der Stahllamellenbeschaffenheit auf das Einlaufverhalten sind in der Tendenz erkennbar, allerdings unterliegen diese starken Wechselwirkungen mit Reibbelag und Schmierstoff. In Versuchen mit Reibbelag LSD-A und Schmierstoff LSD-I zeigen Stahllamellen BCI-St2 höhere Werte $\overline{\mathrm{EW}}$ als Stahllamellen BCI-St1. Im Mittel ist dies tendenziell auch in Versuchen mit Reibbelag LSD-A und Schmierstoff LSD-II erkennbar. Mit Reibbelag LSD-B und Schmierstoff LSD-II tritt hingegen ein gegenteiliges Verhalten auf: hier weisen Versuche mit Stahllamellen BCISt1 höhere Werte $\overline{\mathrm{EW}}$ auf als Stahllamellen BCI-St2. In Kombination mit dem anderen Schmierstoff ist im Mittel die gleiche Tendenz erkennbar.

In den Versuchen mit Kupplungen der Baugröße BCII zeigen sich ebenfalls Einflüsse von Schmierstoff, Reibbelag und Stahllamellenbeschaffenheit auf das Einlaufverhalten charakterisiert durch den Kennwert $\overline{\mathrm{EW}}$.

Tab. 4 fasst die Einflüsse des Reibsystems auf das Einlaufverhalten zusammen.

Einflüsse des Reibsystems auf das Einlaufverhalten sind somit sehr komplex und von deutlichen Wechselwirkungen geprägt.

\section{Diskussion}

Die Methodik „Einlaufverhalten Lamellenkupplungen“ wird herangezogen, um Einflüsse des Reibsystems bestehend aus Stahllamelle, Reibbelag und Schmierstoff auf das Einlaufverhalten systematisch zu untersuchen. Es kommen unterschiedliche Kupplungen (Baugrößen BCI und BCII gemäß Abschn. 2.3) mit verschiedenen Stahllamellenvarianten, Reibbelägen und Schmierstoffen zum Einsatz.

Es zeigt sich kein für alle Baugrößen und Beanspruchungen dominanter Einfluss eines einzelnen Bestandteils des Reibsystems. Zudem sind die Einflüsse von starken Wechselwirkungen geprägt. In Versuchen mit Baugröße BCI sind Einflüsse des Schmierstoffs dominant, mit Baugröße BCII zeigt die Stahllamellenbeschaffenheit die deutlichsten Einflüsse auf das Einlaufverhalten (vgl. Abschn. 3). Hierbei ist zu beachten, dass die Versuche im gleichen Lastkollektiv und unter Verwendung identischer Schmierstoffe sowie nominell gleicher Reibbeläge und Stahllamellen vergleichbarer Fertigungsverfahren durchgeführt werden. Topographische Vermessungen in [12] zeigen allerdings, dass die Beschaffenheit der Stahl- und Belaglamellen trotz gleicher Bezeichnungen deutlich voneinander abweichen.

Eine Systembetrachtung ist somit von zentraler Bedeutung. Soll das Einlaufverhalten einer Kupplung charakterisiert werden, so ist dieses für relevante Lastniveaus an der Kupplung im Komponenten- oder Getriebeversuch zu ermitteln. Die im Rahmen dieser Veröffentlichung dargelegten Untersuchungen ergeben eindeutig, dass Aussagen zum
Einlaufverhalten trotz identischer Schmierstoffe und nominell gleicher Reibbeläge und Stahllamellenvarianten nicht ungeprüft auf andere Kupplungen (andere Bauart, Baugröße, usw.) übertragbar sind. Eine Charakterisierung des Einlauf- und Reibungsverhaltens sollte also stets an der in der Anwendung vorliegenden Kupplung erfolgen.

\section{Zusammenfassung}

Die Methodik „Einlaufverhalten Lamellenkupplungen“ realisiert die Charakterisierung des Einlaufverhaltens nasslaufender Lamellenkupplungen. Im vorliegenden Beitrag werden die Versuchs- und Auswertemethoden zur Charakterisierung des Einlaufverhaltens nasslaufender Lamellenkupplungen im Schlupfbetrieb dargestellt. Mit dem Ziel, das Einlaufverhalten auf mehreren anwendungsnahen Lastniveaus $\mathrm{zu}$ charakterisieren, erfolgt die experimentelle Untersuchung des Reibungsverhaltens in einem Lastkollektiv. Die Auswertung des Einlaufverhaltens basiert auf der Betrachtung von Reibcharakteristiken und neu definierten Reibungszahlkennwerten $\mu_{\text {top }}$ (Reibungszahlniveau) und $\mathrm{m}_{\mu}$ (Reibschwingneigung). Diese werden in Trendverläufen über der Schaltungszahl aufgetragen; die Phase des Einlaufs, gekennzeichnet durch einen nichtlinearen Trendverlauf, wird iterativ mittels Regressionsanalyse identifiziert.

Neu entwickelte Kennwerte zum Einlaufverhalten quantifizieren die Dauer des Einlaufs $\mathrm{x}_{\operatorname{lin}}$ und die maximale Reibungszahländerung im Einlauf $\Delta \mu_{\text {start. }}$ In Gegenüberstellung mehrerer Reibsysteme liefert der Kennwert EW eine Einlauf-Systembewertung.

Anhand dieses Kennwerts wird das Einlaufverhalten von Kupplungen in zwei Baugrößen unter Variation des Reibsystems - je zwei Reibbeläge, Stahllamellenvarianten und Schmierstoffe - quantifiziert gegenübergestellt. Es zeigt sich kein für alle Baugrößen und Beanspruchungen dominanter Einfluss eines einzelnen Bestandteils des Reibsystems. Zudem sind die Einflüsse von starken Wechselwirkungen geprägt. Für eine Charakterisierung des Einlaufverhaltens ist somit eine Systembetrachtung unbedingt erforderlich.

Danksagung Die vorgestellten Erkenntnisse basieren auf dem Forschungsvorhaben FVA-Nr. 343/IV; gefördert aus Eigenmitteln der Forschungsvereinigung Antriebstechnik e. V. (FVA). Die Autoren bedanken sich für die Förderung und Unterstützung bei der FVA und den Mitgliedern des projektbegleitenden Ausschusses.

Danksagung The authors would like to thank for sponsorship and support received from the Research Association for Drive Technology e. V. (FVA) and the members of the project committee.

Funding The presented results are based on the research project FVA no. 343/IV undertaken by the Research Association for Drive Technology e. V. (FVA) 
Funding Open Access funding enabled and organized by Projekt DEAL.

Interessenkonflikt K. Völkel, H. Pflaum und K. Stahl geben an, dass kein Interessenkonflikt besteht.

Open Access Dieser Artikel wird unter der Creative Commons Namensnennung 4.0 International Lizenz veröffentlicht, welche die Nutzung, Vervielfältigung, Bearbeitung, Verbreitung und Wiedergabe in jeglichem Medium und Format erlaubt, sofern Sie den/die ursprünglichen Autor(en) und die Quelle ordnungsgemäß nennen, einen Link zur Creative Commons Lizenz beifügen und angeben, ob Änderungen vorgenommen wurden.

Die in diesem Artikel enthaltenen Bilder und sonstiges Drittmaterial unterliegen ebenfalls der genannten Creative Commons Lizenz, sofern sich aus der Abbildungslegende nichts anderes ergibt. Sofern das betreffende Material nicht unter der genannten Creative Commons Lizenz steht und die betreffende Handlung nicht nach gesetzlichen Vorschriften erlaubt ist, ist für die oben aufgeführten Weiterverwendungen des Materials die Einwilligung des jeweiligen Rechteinhabers einzuholen.

Weitere Details zur Lizenz entnehmen Sie bitte der Lizenzinformation auf http://creativecommons.org/licenses/by/4.0/deed.de.

\section{Literatur}

1. Rao G (2011) Modellierung und Simulation des Systemverhaltens nasslaufender Lamellenkupplungen. Dissertation, Technische Universität Dresden

2. Ingram M, Spikes H, Noles J et al (2010) Contact properties of a wet clutch friction material. Tribol Int. https://doi.org/10.1016/j. triboint.2009.11.008

3. Stockinger U, Mühlenstrodt K, Voelkel K et al (2019) Analyse tribologischer Schichten mit der Flugzeit-Sekundärionenmassenspektrometrie (ToF-SIMS) - Additiveinflüsse auf das Reibungsverhalten nasslaufender Lamellenkupplungen. Forsch Ingenieurwes 83:219-226. https://doi.org/10.1007/s10010-019-00305-0
4. Völkel K, Rothemund M, Albarracin Garibello S et al (2019) On the simulation of the micro-contact of rough surfaces using the example of wet friction clutch materials. Lubricants. https://doi.org/10.3390/ lubricants 7050041

5. Sittig K (2007) Tribologisch induzierte oberflächennahe Veränderungen der Stahl- und Belaglamellen einer nasslaufenden Lamellenkupplung. Dissertation, Universität Karlsruhe

6. Kitahara S, Matsumoto T (1996) The relationship between porosity and mechanical strength in paper-based friction materials. SAE Technical Paper 960982. https://doi.org/10.4271/960982

7. Lorentz B, Albers A (2013) A numerical model for mixed lubrication taking into account surface topography, tangential adhesion effects and plastic deformations. Tribol Int. https://doi.org/10.1016/ j.triboint.2012.08.023

8. Bäse MU, Dzimko M, Deters L (2016) Empirische Bewertung von Zusammenhängen zwischen Endbearbeitungsparametern und Reibungsverhalten von Lamellenreibpaarungen im Mikroschlupfbetrieb. In: Gesellschaft für Tribologie e. V. (Hrsg) 57. TribologieFachtagung, Bd. 2. GfT Gesellschaft für Tribologie e. V., Aachen (Art. 25)

9. Katsukawa M (2019) Effects of the physical properties of resins on friction performance. SAE Technical Paper Series. SAE, Warrendale

10. Nyman P, Mäki R, Olsson R et al (2006) Influence of surface topography on friction characteristics in wet clutch applications. Wear 261:46-52. https://doi.org/10.1016/j.wear.2005.09.020

11. Zou Q, Rao C, Barber G et al (2013) Investigation of surface characteristics and tribological behavior of clutch plate materials. Wear 302:1378-1383

12. Völkel K (2020) Charakterisierung des Einlaufverhaltens nasslaufender Lamellenkupplungen. Dissertation, TU München

13. Völkel K, Pflaum H, Stahl K (2019) Einflüsse der Stahllamelle auf das Einlaufverhalten von Lamellenkupplungen. Forsch Ingenieurwes 28:2148. https://doi.org/10.1007/s10010-019-00303-2

14. Völkel K, Pflaum H, Stahl K (2019) 343/IV - Einlaufverhalten Stahllamelle II: Sachstandsberichte. FVA, Frankfurt 